\title{
Distribution Sensitivity for Certain Classes of Chance-Constrained Models with Application to Power Dispatch ${ }^{1,2}$
}

\author{
W. RÖMISCH ${ }^{3}$ AND R. SCHULtz ${ }^{4}$ \\ Communicated by A. V. Fiacco
}

\begin{abstract}
Using results from parametric optimization, we derive for chance-constrained stochastic programs quantitative stability properties for locally optimal values and sets of local minimizers when the underlying probability distribution is subjected to perturbations in a metric space of probability measures. Emphasis is placed on verifiable sufficient conditions for the constraint-set mapping to fulfill a Lipschitz property which is essential for the stability results. Both convex and nonconvex problems are investigated. For a chance-constrained model of power dispatch, where the power demand enters as a random vector with incompletely known probability distribution, we discuss consequences of our general results for the stability of optimal generation costs and optimal generation policies.
\end{abstract}

Key Words. Parametric optimization, chance-constrained stochastic programming, sensitivity analysis, optimal power dispatch.

\section{Introduction}

To ensure a certain level of reliability for the solutions to optimization problems containing random data, it has become an accepted approach to

\footnotetext{
${ }^{1}$ The authors thank P. Kleinmann (formerly with the Humboldt-Universität, Berlin, Germany) for his active cooperation in designing the power dispatch model and J. Mayer (MTA SZTAKI, Budapest, Hungary) for his insight into energy optimization. Further thanks are due to the referees for their constructive criticism.

${ }^{2}$ This research was developed in the course of a contract study between the International Institute for Applied Systems Analysis, Laxenburg, Austria and the Humboldt-Universität, Berlin, Germany.

${ }^{3}$ Associate Professor, Humboldt-Universität, Sektion Mathematik, Berlin, Germany.

${ }^{4}$ Research Assistant, Humboldt-Universität, Sektion Mathematik, Berlin, Germany.
} 
introduce probabilistic (or chance) constraints into the model. This leads to the following type of optimization problems:

$$
\min \left\{f(x): x \in \mathbb{R}^{m}, \mu\left(\left\{z \in \mathbb{R}^{s}: x \in X(z)\right\}\right) \geq p_{0}\right\},
$$

where $f$ is a real-valued function defined on $\mathbb{R}^{m}, X$ is a set-valued mapping from $\mathbb{R}^{s}$ into $\mathbb{R}^{m}, p_{0} \in(0,1)$ is a prescribed probability level, and $\mu$ is a probability distribution on $\mathbb{R}^{s}$. For basic results on chance-constrained problems, consult Refs. 1 and 2 and the references therein.

We are going to study the behavior of (1) with respect to small perturbations of the probability distribution $\mu$. This is motivated by the fact that, in practice, one is often faced with incomplete information on the underlying probability distributions. Therefore, applicable models should at least enjoy some kind of stability with respect to variations of the distributions.

Our approach relies on stability results for parametric optimization problems with parameters in metric spaces (see Ref. 3 for quantitative aspects and Refs. 4 and 5 for qualitative aspects). As the parameter space, we consider the space $\mathscr{P}\left(\mathbb{R}^{s}\right)$ of all Borel probability measures on $\mathbb{R}^{s}$ equipped with a suitable metric. We are aiming at quantitative continuity properties for the mappings assigning to each parameter the local optimal value and the set of local minimizers, respectively.

Because of its central place in the convergence theory for probability measures, it seems appropriate to study stability with respect to the topology of weak convergence on $\mathscr{P}\left(\mathbb{R}^{s}\right)$. This has been done in Ref. 6, using the results of Ref. 5, and in Ref. 7. An example in Ref. 8 indicates that stability of (1) with respect to the topology of weak convergence cannot be expected in general without additional smoothness assumptions on the measure $\mu$. It turned out in Refs. 8-10 that the so called $\mathscr{B}$-discrepancy (this notion is made precise in Section 2) is a suitable metric on $\mathscr{P}\left(\mathbb{R}^{5}\right)$ for the sensitivity analysis of (1). Compared to Refs. 8-10, the present paper deals with more practicable models, and it gives sufficient conditions for the also quantitative stability of optimal values and optimal solutions which are easier to verify. For quite a large class of distributions, we obtain in Section 2 the upper semicontinuity of the optimal-set mapping and the Lipschitz continuity of the optimal value function. We show that, under more restrictive assumptions, it is possible to quantify also the upper semicontinuity of the optimal-set mapping.

For probability distributions which depend on Euclidean parameters, the sensitivity of optimal solutions to chance-constrained problems has been studied in Ref. 11 using an approach via the implicit function theorem (Ref. 12). Stability in chance-constrained programming is studied also in Refs. 13 and 14. Whereas the results of Ref. 13 are relevant for approximation schemes, Ref. 14 deals with a statistical approach. 
The general results that we obtain in Section 2 are specified in two directions: First, at the end of Section 2, we briefly outline potential applications in the situation where the distribution $\mu$ is completely unknown and, therefore, estimated via empirical measures. The second specification, which is the topic dealt with in Section 3, concerns an optimal power-dispatch model.

The investigations in Section 3 serve to illustrate our general results and to discuss the aspect of stability when incorporating chance constraints into power-dispatch models. For more details about power dispatch, we have to refer to the literature (Refs. 15-23).

Next, we introduce some basic concepts and notations which are used throughout. For $v \in \mathscr{P}\left(\mathbb{R}^{s}\right)$, we denote by $F_{v}$ the distribution function of $v$ and set, for $p \in[0,1]$,

$$
C_{p}(v):=\left\{x \in \mathbb{R}^{m}: v\left(X^{-}(x)\right) \geq p\right\} .
$$

Hence, problem (1) becomes

$$
\min \left\{f(x): x \in C_{p_{0}}(\mu)\right\} .
$$

Given $V \subseteq \mathbb{R}^{m}$ and $v \in \mathscr{P}\left(\mathbb{R}^{s}\right)$, we denote

$$
\begin{aligned}
& \varphi_{V}(v):=\inf \left\{f(x): x \in C_{p_{0}}(v) \cap \mathrm{cl} V\right\}, \\
& \psi_{V}(v):=\left\{x \in C_{p_{0}}(v) \cap \mathrm{cl} V: f(x)=\varphi_{V}(v)\right\},
\end{aligned}
$$

where we employ the abbreviation cl for closure. Following Refs. 5 and 3 , we call a nonempty subset $M$ of $\mathbb{R}^{m}$ a complete local minimizing (CLM) set for (1) with respect to $Q$ if $Q$ is an open subset of $\mathbb{R}^{m}$ such that $Q \supset M$ and $M=\psi_{Q}(\mu)$. Later on, we will briefly say that $\psi_{Q}(\mu)$ is a CLM set for (1), which means that the set in question is a CLM set for (1) with respect to $Q$. Examples of CLM sets are the set of global minimizers [which we shall denote by $\psi(\mu)$ and, accordingly, the global optimal value by $\varphi(\mu)$ ] or strict local minimizing points. We call a multifunction $\Gamma$ from a metric space $(T, d)$ to $\mathbb{R}^{m}$ closed at $t_{0} \in T$ if $t_{k} \rightarrow t_{0}, x_{k} \rightarrow x_{0}, x_{k} \in \Gamma\left(t_{k}\right), k \in \mathbb{N}$, imply $x_{0} \in \Gamma\left(t_{0}\right) ; \Gamma$ is said to be upper semicontinuous (usc) at $t_{0} \in T$ if, for any open set $G \supset \Gamma\left(t_{0}\right)$, there exists a neighborhood $U$ of $t_{0}$ such that $\Gamma(t) \subset G$ whenever $t \in U$; and $\Gamma$ is said to be pseudo-Lipschitzian at $\left(x_{0}, t_{0}\right) \in \Gamma\left(t_{0}\right) \times T$ (cf. Ref. 24) if there are neighborhoods $U$ and $V$ of $t_{0}$ and $x_{0}$, respectively, and a constant $L>0$ such that

$$
\Gamma(t) \cap V \subseteq \Gamma(\tilde{t})+L d(t, \tilde{t}) B_{m}, \quad \text { whenever } t, \tilde{t} \in U,
$$

where $B_{m}$ is the closed unit ball in $\mathbb{R}^{m}$. For $x_{0} \in \mathbb{R}^{m}$ and $\epsilon>0$, we denote

$$
B\left(x_{0}, \epsilon\right):=\left\{x \in \mathbb{R}^{m}:\left\|x-x_{0}\right\| \leq \epsilon\right\} .
$$

Thus, $B_{m}=B(0,1)$. Here, $\|\cdot\|$ is the Euclidean norm on $\mathbb{R}^{m}$. 


\section{Sensitivity Analysis}

To complete the prerequisites for our sensitivity analysis, we specify the metric in the parameter space $\mathscr{P}\left(\mathbb{R}^{s}\right)$ as follows: Consider the $\mathscr{B}$-discrepancy

$$
\alpha_{\mathscr{B}}(\mu, v):=\sup \{|\mu(B)-v(B)|: B \in \mathscr{B}\}, \quad \mu, v \in \mathscr{P}\left(\mathbb{R}^{s}\right),
$$

where $\mathscr{B}$ is a proper subclass of Borel sets in $\mathbb{R}^{s}$, in the sense that it is chosen such that $\alpha_{\mathscr{B}}$ forms a metric (i.e., $\mathscr{B}$ is a determining class, Ref. 25 ) and that it contains all the preimages $X^{-}(x):=\left\{z \in \mathbb{R}^{s}: x \in X(z)\right\}, x \in \mathbb{R}^{m}$; cf. (1).

Our first theorem asserts in a fairly general frame sensitivity properties for solutions of a parametric chance-constrained problem. The proof, which relies on stability results for abstract parametric programming problems obtained by Klatte in Ref. 3, can be found in Ref. 10 (Theorem 5.4).

Theorem 2.1. Let in (1)

$$
\mu \in \mathscr{P}\left(\mathbb{R}^{s}\right), \quad p_{0} \in(0,1), \quad\left\{X^{-}(x): x \in \mathbb{R}^{m}\right\} \subseteq \mathscr{B} .
$$

Let further $X$ be a closed multifunction; and let $f$ be Lipschitzian on bounded sets. Assume that there exists a bounded open set $V \subset \mathbb{R}^{m}$ such that $\psi_{V}(\mu)$ is a CLM set for (1). Let the multifunction $p \mapsto C_{p}(\mu)$ be pseudo-Lipschitzian at each $\left(x_{0}, p_{0}\right) \in \psi_{V}(\mu) \times\left\{p_{0}\right\}$. Then, $\psi_{V}$ is use at $\mu$ with respect to the metric $\alpha_{\mathscr{B}}$ on $\mathscr{P}\left(\mathbb{R}^{s}\right)$, and there exist constants $L>0$ and $\delta>0$ such that

$$
\psi_{\nu}(v) \text { is a CLM set for (1) }
$$

and

$$
\left|\varphi_{V}(\mu)-\varphi_{V}(v)\right| \leq L \alpha_{\mathscr{B}}(\mu, v),
$$

whenever $\alpha_{\mathscr{B}}(\mu, v)<\delta, v \in \mathscr{P}\left(\mathbb{R}^{s}\right)$.

As in Ref. 3, it is possible to quantify the upper semicontinuity of the solution-set mapping when imposing more restrictive assumptions. So, the following result was inspired by the material in Ref. 3, although there is the difference that the result also covers the situation when the solution set of the unperturbed problem is not a singleton.

Theorem 2.2. Let $\mu, p_{0}, X, \mathscr{B}$, and $f$ be as in Theorem 2.1. Additionally to the assumptions of Theorem 2.1, let there exist $x_{0} \in C_{p_{0}}(\mu)$ and constants $c>0, q \geq 1$ such that, for all $x \in C_{p_{0}}(\mu) \cap \mathrm{cl} V$, we have

$$
f(x) \geq f\left(x_{0}\right)+c \| x-\left.x_{0}\right|_{*} ^{q},
$$


where $\| \cdot l_{*}$ is a nontrivial seminorm on $\mathbb{R}^{m}$. Then, there exist $L>0$ and $\delta>0$ such that

$$
\psi_{v}(v) \text { is a CLM set for (1) }
$$

and

$$
\begin{aligned}
& \| x-\left.x_{0}\right|_{*} ^{q} \leq L \alpha_{\mathscr{F}}(\mu, v), \text { for all } x \in \psi_{\nu}(v), \\
& \text { whenever } \alpha_{\mathscr{B}}(\mu, v)<\delta, v \in \mathscr{P}\left(\mathbb{R}^{s}\right) .
\end{aligned}
$$

Proof. By assumption, $\psi_{V}(\mu)$ is compact. Since $p \mapsto C_{p}(\mu)$ is pseudom Lipschitzian at each $\left(x_{0}, p_{0}\right) \in \psi_{V}(\mu) \times\left\{p_{0}\right\}$, this together with Proposition 5.3 in Ref. 10 and a corresponding construction in Ref. 3 implies that there are open neighborhoods $V_{0}$ of $\psi_{V}(\mu), U_{0}$ of $\mu$, and a constant $L_{0}>0$ such that

$$
\begin{aligned}
& \psi_{V}(\mu) \subset V_{0} \subseteq V \text { and } \\
& C_{p_{0}}(v) \cap V_{0} \subseteq C_{p_{0}}(\mu)+L_{0} \alpha_{\mathscr{B}}(\mu, v) B_{m}, \\
& C_{p_{0}}(\mu) \cap V_{0} \subseteq C_{p_{0}}(v)+L_{0} \alpha_{\mathscr{B}}(\mu, v) B_{m}, \text { whenever } v \in U_{0} .
\end{aligned}
$$

Now, select an open set $V_{1}$ and a constant $\rho>0$ such that

$$
\psi_{V}(\mu) \subset V_{1}, \quad V_{1}+B(0, \rho) \subseteq V_{0}
$$

By Theorem 2.1, the mapping $\psi_{V}$ is usc at $\mu$; hence, there exists a constant $\delta_{0}>0$ such that

$$
\psi_{V}(v) \subset V_{1}, \text { whenever } \gamma \in \mathscr{P}\left(\mathbb{R}^{s}\right), \alpha_{\mathscr{B}}(\mu, v)<\delta_{0}
$$

We choose $\delta>0$ such that $\delta \leq \min \left\{1, \delta_{0}, \rho / L_{0}, \delta_{\phi}\right\}$ and $\left\{v \in \mathscr{P}\left(\mathbb{R}^{s}\right)\right.$ : $\left.\alpha_{\mathscr{A}}(\mu, v)<\delta\right\} \leq U_{0} ; \delta_{\varphi}$ denotes the bound on $\alpha_{\mathscr{g}}(\mu, v)$ arising in the Lipschitz property for $\varphi_{V}$ which holds due to Theorem 2.1 .

Let $v \in \mathscr{P}\left(\mathbb{R}^{s}\right)$ such that $\alpha_{\mathscr{R}}(\mu, v)<\delta$ and $x \in \psi_{V}(v)$. Then, we have $x \in C_{p_{0}}(v) \cap V_{0}$; and by (2), there exists $\vec{x} \in C_{p_{0}}(\mu)$ such that

$$
\|x-\bar{x}\| \leq L_{0} \alpha_{\mathscr{B}}(\mu, v) .
$$

This yields

$$
\|x-\bar{x}\|<L_{0} \delta \leq \rho
$$

implying that

$$
\bar{x} \in C_{p_{0}}(\mu) \cap V_{0} \subseteq C_{p_{0}}(\mu) \cap \mathrm{dl} V .
$$


Hence,

$$
f(x) \geq f(\bar{x})-L_{f}\|x-\bar{x}\| \geq f\left(x_{0}\right)+c \| \bar{x}-\left.x_{0}\right|_{*} ^{q}-L_{f} L_{0} \alpha_{\mathscr{R}}(\mu, v),
$$

where $L_{f}$ is a Lipschitz constant for $f$ on $\mathrm{cl} V$. This yields

$$
\begin{aligned}
\| \bar{x}-\left.x_{0}\right|_{*} ^{q} & \leq(1 / c)\left[\left(f(x)-f\left(x_{0}\right)\right)+L_{f} L_{0} \alpha_{\mathscr{B}}(\mu, v)\right] \\
& \leq(1 / c)\left[\left|\varphi_{V}(\mu)-\varphi_{V}(v)\right|+L_{f} L_{0} \alpha_{\mathscr{B}}(\mu, v)\right] .
\end{aligned}
$$

In view of the Lipschitz property for $\varphi_{V}$ (cf. Theorem 2.1) and the inequality

$$
\left\|x-\left.x_{0}\right|_{*} \leq|| x-\left.\bar{x}\right|_{*}+\right\| \bar{x}-\left.x_{0}\right|_{*}
$$

it suffices to estimate $\| x-\left.\bar{x}\right|_{*}$ to prove the assertion. As a general property for the seminorm $\|\left.\cdot\right|_{*}$, we have that there exists a constant $c_{1}>0$ such that,

$$
\left\|\left.y\right|_{*} \leq c_{1}\right\| y \|, \quad \text { for all } y \in \mathbb{R}^{m}
$$

Therefore, by (3),

$$
\left\|x-\left.\bar{x}\right|_{*} \leq c_{1}\right\| x-\bar{x} \| \leq c_{1} L_{0} \alpha_{\text {保 }}(\mu, v)
$$

which completes the proof.

Remark 2.1. The above results may also be viewed as stability results with respect to perturbations of $\mu$ in the space $\mathscr{P}\left(\mathbb{R}^{s}\right)$ equipped with the topology of weak convergence if $\mathscr{B}$ is a $\mu$-uniformity class of Borel sets in $\mathbb{R}^{s}$. Recall that $\mathscr{B}$ is a $\mu$-uniformity class if $\alpha_{\mathscr{B}}\left(\mu_{n}, \mu\right) \rightarrow 0$ holds for every sequence $\left(\mu_{n}\right)$ converging weakly to $\mu$ (Ref. 26 ). If $\mathscr{B}$ is a subclass of

$$
\mathscr{B}_{C}:=\left\{B \subset \mathbb{R}^{s}: B \text { is convex and Borel }\right\} \text {, }
$$

the following result is known (Theorem 2.11 in Ref. 26 ): $\mathscr{B}$ is a $\mu$-uniformity class iff $\mu(\partial B)=0$ for all $B \in \mathscr{B}$; here, $\partial B$ denotes the topological boundary of $B$. Hence, the class

$$
\mathscr{B}_{R}:=\left\{\varnothing,(-\infty, z]: z \in \mathbb{R}^{s}\right\}
$$

is a $\mu$-uniformity class if the distribution function $F_{\mu}$ of $\mu$ is continuous on $\mathbb{R}^{s}$, and $\mathscr{B}_{C}$ is a $\mu$-uniformity class if $\mu$ has a density with respect to Lebesgue measure on $\mathbb{R}^{s}$. We note that

$$
d_{K}(\mu, v):=\alpha_{B_{R}}(\mu, v)=\sup _{z \in \mathbb{R}^{s}}\left|F_{\mu}(z)-F_{v}(z)\right|
$$

is the so-called Kolmogorov distance on $\mathscr{P}\left(\mathbb{R}^{s}\right)$. 
We now reveal conditions on the measure $\mu$ and on the multifunction $X$ to have the mapping $p \mapsto C_{p}(\mu)$ pseudo-Lipschitzian at some point $\left(x_{0}, p_{0}\right) \in \mathbb{R}^{m} \times[0,1]$, thus arriving at stability results which are specifications of Theorems 2.1 and 2.2. The first part of our analysis concerns the special case where the sets $C_{p}(\mu), p \in[0,1]$, are convex.

We say that $\mu \in \mathscr{P}\left(\mathbb{R}^{s}\right)$ belongs to the class $\mathscr{M}_{r}, r \in[-\infty,+\infty)$, if for all $\lambda \in[0,1]$ and all Borel sets $B_{1}, B_{2} \subset \mathbb{R}^{s}$, such that $\lambda B_{1}+(1-\lambda) B_{2}$ is Borel,

$$
\mu\left(\lambda B_{1}+(1-\lambda) B_{2}\right) \geq\left\{\lambda\left[\mu\left(B_{1}\right)\right]^{r}+(1-\lambda)\left[\mu\left(B_{2}\right)\right]^{r}\right\}^{1 / r} .
$$

Here,

$$
\lambda B_{1}+(1-\lambda) B_{2}:=\left\{\lambda b_{1}+(1-\lambda) b_{2}: b_{i} \in B_{i}, i=1,2\right\} .
$$

In the cases $r=0$ and $r=-\infty$, the right-hand side of (4) is interpreted by continuity as

$$
\left[\mu\left(B_{1}\right)\right]^{\lambda}\left[\mu\left(B_{2}\right)\right]^{1-\lambda} \text { and } \min \left\{\mu\left(B_{1}\right), \mu\left(B_{2}\right)\right\},
$$

respectively. The classes $\mathscr{M}_{r}$ have been introduced and studied in Refs. 27 29. Clearly, we have

$$
\mathscr{M}_{r_{1}} \supseteq \mathscr{M}_{r_{2}},-\infty \leq r_{1} \leq r_{2}<+\infty .
$$

Measures belonging to $\mathscr{M}_{0}\left(\mathscr{M}_{-\infty}\right)$ are called logarithmic concave (quasiconcave). $\mathscr{M}_{0}$ was first and extensively studied by Prékopa in Refs. 28 and 30. It is known (cf., e.g., Theorem 1 in Ref. 29) that $\mu$ belongs to $\mathscr{M}_{r}, r \in[-\infty, 0]$, if $\mu$ has a density $f_{\mu}$ and $f_{\mu}^{\tau /(1-r s)}$ is convex, $-\infty \leq r<0, \log f_{\mu}$ is concave $(r=0)$.

It is well known that the nondegenerate multivariate normal, the multivariate beta, Dirichlet and Wishart, a special multivariate gamma, and the multivariate Pareto $t$ and $F$ distributions (cf. Ref. 31) belong to $\mathscr{M}$, for some $r \leq 0$ (see Refs. 27-30).

For convex chance constraints, we now have the following corollary to Theorem 2.1.

Corollary 2.1. Assume that in (1) $\mu \in \mathscr{A}_{r}$ for some $r \in(-\infty, 0]$, $p_{0} \in(0,1), X$ has a closed convex graph, and $f$ is Lipschitzian on bounded sets. Let $\left\{X^{-}(x): x \in \mathbb{R}^{m}\right\} \subseteq \mathscr{B} \subseteq \mathscr{B}_{C}$ and $\bigcup_{z \in \mathbb{R}^{s}} X(z)$ be bounded. Assume that there exists $\bar{x} \in \mathbb{R}^{m}$ such that $\mu\left(X^{-}(\bar{x})\right)>p_{0}$ (Slater condition). Then, $\psi$ is usc at $\mu$ with respect to $\alpha_{\mathscr{B}}$ on $\mathscr{P}\left(\mathbb{R}^{s}\right)$ and there exist constants $L>0$ and $\delta>0$ such that $\psi(v) \neq \varnothing$ and $|\varphi(\mu)-\varphi(v)| \leq L \alpha_{\mathscr{Q}}(\mu, v)$, whenever $\alpha_{\mathscr{B}}(\mu, v)<\delta, v \in \mathscr{P}\left(\mathbb{R}^{S}\right)$. 
Proof. Since $\mathscr{M}_{0} \subseteq \mathscr{M}_{r}$ for each $r \in(-\infty, 0)$, we assume w.l.o.g. that $r \in(-\infty, 0)$ and write $(1)$ in the equivalent form

$$
\min \left\{f(x): x \in \mathbb{R}^{m},\left[\mu\left(X^{-}(x)\right)\right]^{r} \leq p_{0}^{r}\right\} .
$$

Since the constraint set of (5) is closed (see Ref. 10) and bounded (according to the assumptions), we have that the set of global minimizers $\psi(\mu)$ to (5) is nonempty and that the assumptions in Theorem 2.1 concerning the CLM set may be fulfilled with a bounded open set $V \supset \bigcup_{z \in \mathbb{R}^{s}} X(z)$; hence, the mappings $\psi$ and $\psi_{V}, \varphi$ and $\varphi_{V}$ coincide.

We define the function $g(x):=\left[\mu\left(X^{-}(x)\right)\right]^{r}$ from $\mathbb{R}^{m}$ to $(-\infty, \infty]$ and have, for all $x_{1}, x_{2} \in \mathbb{R}^{m}$ and $\lambda \in[0,1]$, that

$$
\begin{aligned}
g\left(\lambda x_{1}+(1-\lambda) x_{2}\right) & =\left[\mu\left(X^{-}\left(\lambda x_{1}+(1-\lambda) x_{2}\right)\right)\right]^{r} \\
& \leq\left[\mu\left(\lambda X^{-}\left(x_{1}\right)+(1-\lambda) X^{-}\left(x_{2}\right)\right)\right]^{r} \\
& \leq \lambda\left[\mu\left(X^{-}\left(x_{1}\right)\right)\right]^{r}+(1-\lambda)\left[\mu\left(X^{-}\left(x_{2}\right)\right)\right]^{r} .
\end{aligned}
$$

Here, we used in the first inequality that $X$ has convex graph, and in the second that (4) is valid.

Hence, $g$ is convex and the multifunction $\Gamma$ (from $\mathbb{R}$ to $\mathbb{R}^{m}$ ) defined by

$$
\Gamma(t):=\left\{x \in \mathbb{R}^{m}: g(x) \leq t\right\}, \quad t \in \mathbb{R},
$$

has a closed convex graph. Due to Theorem 2 in Ref. $32, \Gamma$ is pseudoLipschitzian at each $\left(x_{0}, t_{0}\right)$ with $x_{0} \in \Gamma\left(t_{0}\right)$ and $t_{0}$ belonging to the interior of $\{t \in \mathbb{R}: \Gamma(t) \neq \varnothing\}$. Since $g(\bar{x})<p_{0}^{r}, p_{0}^{r}$ is an interior point of $\{t \in \mathbb{R}: \Gamma(t) \neq \varnothing\}$. Therefore, $\Gamma$ is pseudo-Lipschitzian at $\left(x_{0}, p_{0}^{r}\right)$ for each $x_{0} \in \Gamma\left(p_{0}^{r}\right)$. In view of $C_{p}(\mu)=\Gamma\left(p^{r}\right)$, this means that there exist positive constants $L, \delta$ and a neighborhood $V$ of $x_{0} \in C_{p_{0}}(\mu)$ such that

$$
C_{p}(\mu) \cap V \subseteq C_{\tilde{p}}(\mu)+L\left|p^{r}-\tilde{p}^{r}\right| B_{m},
$$

whenever $p^{r}, \tilde{p}^{r} \in B\left(p_{0}^{r}, \delta\right)$. Since the function $\xi \mapsto \xi^{r}$ is locally Lipschitzian for positive $\xi$, we obtain that the multifunction $p \mapsto C_{p}(\mu)$ is pseudo-Lipschitzian at each $\left(x_{0}, p_{0}\right) \in C_{p_{0}}(\mu) \times\left\{p_{0}\right\}$. The assertion now follows from Theorem 2.1.

The above corollary extends results obtained by Salinetti (Corollary 3.2.2 in Ref. 13) and Wang (Theorem 6 in Ref. 7).

We remark that the Lipschitz modulus $L$ in Corollary 2.1 can be estimated above, povided that $\delta$ [which restricts $\alpha_{\mathscr{B}}(\mu, v)$ ] is sufficiently small. According to Ref, 3 , such a bound for $L$ is given by $L_{f}\left(L_{C}+1\right)$, where $L_{f}$ is the Lipschitz modulus for $f$ on $\mathrm{cl} V$ (cf. the above proof) and $L_{C}$ is the 
modulus that we have for $p \mapsto C_{p}(\mu)$, since it is pseudo-Lipschitzian (cf. the proofs of Proposition 5.3 and Theorem 5.4 in Ref. 10). Starting from results of, for example, Robinson (Theorem 2 in Ref. 32) or Pshenichny (Ref. 33, Theorem 1.2, p. 100), a further estimation of $L_{C}$ is possible. This exploits the uniform compactness of the sets $C_{p}(\mu), p \in(0,1)$, and the explicit knowledge of the Slater point $\bar{x}$.

Remark 2.2. Let, additionally to the assumptions of Corollary 2.1, there exist $x_{0} \in C_{p_{0}}(\mu)$ and $c>0$ such that

$$
f(x) \geq f\left(x_{0}\right)+c \| x-\left.x_{0}\right|_{*} ^{q}, \quad \text { for all } x \in C_{p_{0}}(\mu),
$$

where $\|\left.\cdot\right|_{*}$ is a nontrivial seminorm on $\mathbb{R}^{m}$. Then, using Theorem 2.2 , we arrive at the following quantitative stability result for the global minimizers:

There exist constants $L>0$ and $\delta>0$ such that

$\| x-\left.x_{0}\right|_{*} ^{q} \leq L \alpha_{\mathscr{B}}(\mu, v)$, for all $x \in \psi(v)$,

whenever $\alpha_{\mathscr{P}}(\mu, \nu)<\delta, v \in \mathscr{P}\left(\mathbb{R}^{s}\right)$.

We proceed with the nonconvex case. Here, we assume that the multifunction $X$ is given by

$$
X(z):=\left\{x \in X_{0}: A x \geq z\right\}, \quad z \in \mathbb{R}^{s},
$$

where $X_{0} \subseteq \mathbb{R}^{m}$ is a nonempty closed set and $A \in L\left(\mathbb{R}^{m}, \mathbb{R}^{s}\right)$. Again, sufficient conditions are essential under which the multifunction $p \mapsto C_{p}(\mu)$ is pseudoLipschitzian at certain points $\left(x_{0}, p_{0}\right)$. From the literature, it is known that constraint qualifications are such sufficient conditions (cf. Refs. 24, 34). As an example of results that can be derived in this way, we present the following proposition.

Proposition 2.1. Let the distribution function $F_{\mu}$ of $\mu \in \mathscr{P}\left(\mathbb{R}^{s}\right)$ be locally Lipschitzian, $p_{0} \in(0,1)$; let $X_{0}$ be a closed set; and let $x_{0} \in X_{0}$ be such that $F_{\mu}\left(A x_{0}\right) \geq p_{0}$. In the case $F_{\mu}\left(A x_{0}\right)=p_{0}$, let further $\partial F_{\mu}\left(A x_{0}\right) \cap N_{X_{0}}\left(x_{0}\right)=\varnothing$, where $\partial$ denotes the Clarke generalized gradient of $F_{\mu}(A \cdot)$ and $N_{X_{0}}\left(x_{0}\right)$ is the Clarke normal cone to $X_{0}$ at $x_{0}$ (see Ref. 35). Then, the multifunction $p \mapsto\left\{x \in X_{0}: F_{\mu}(A x) \geq p\right\}$ is pseudo-Lipschitzian at $\left(x_{0}, p_{0}\right)$.

\section{Proof. Define}

$$
\Gamma(p):=\left\{x: p-F_{\mu}(A x) \leq 0,(p, x) \in \mathbb{R} \times X_{0}\right\} .
$$


According to Theorem 3.2 in Ref. 24, the multifunction $\Gamma$ is pseudo-Lipschitzian at $\left(x_{0}, p_{0}\right)$ if the following holds:

if there are $y, z \in \mathbb{R}$ such that

$$
\begin{aligned}
& y \geq 0, y\left(p_{0}-F_{\mu}\left(A x_{0}\right)\right)=0, \text { and } \\
& (0, z) \in\left\{y(x, 1)+(\tilde{x}, 0):-x \in \partial F_{\mu}\left(A x_{0}\right), \tilde{x} \in N_{X_{0}}\left(x_{0}\right)\right\}, \\
& \text { then } y=z=0 .
\end{aligned}
$$

Now, assume that, in our situation, the above does not hold. Then, there are $y>0, \quad \bar{x} \in \partial F_{\mu}\left(A x_{0}\right)$, and $\tilde{x} \in N_{X_{0}}\left(x_{0}\right)$ such that $-y \bar{x}+\tilde{x}=0$. The last identity, however, implies $\bar{x} \in N_{X_{0}}\left(x_{0}\right)$, which contradicts $\partial F_{\mu}\left(A x_{0}\right) \cap$ $N_{X_{0}}\left(x_{0}\right)=\varnothing$.

Of course, making use of Proposition 2.1 hinges upon whether one is able to check the constraint qualification

$$
\partial F_{\mu}\left(A x_{0}\right) \cap N_{X_{0}}\left(x_{0}\right)=\varnothing .
$$

In applications, this may be a formidable task, especially when exploiting the result in its fullest generality.

Therefore, in the following, we establish by an alternative way sufficient conditions which are easier to verify. These conditions are similar to that given in Ref. 9 and are essentially based on a local growth property for the distribution function of the probability distribution in the unperturbed problem [see (8) below].

Corollary 2.2. In (1), let $\mu \in \mathscr{P}\left(\mathbb{R}^{s}\right)$ have a continuous distribution function $F_{\mu}$; further, let $p_{0} \in(0,1)$, and let the multifunction $X$ be given by (7), where the set $X_{0}$ is convex and closed. Suppose that there exists a bounded open set $V \subset \mathbb{R}^{m}$ such that $\psi_{v}(\mu)$ is a CLM set for (1). For each $x_{0} \in \psi_{\nu}(\mu)$ with $F_{\mu}\left(A x_{0}\right)=p_{0}$, let there exist reals $\epsilon_{0}>0$ and $c>0$ such that, for any $x \in X_{0} \cap B\left(x_{0}, \epsilon_{0}\right)$, there exists $\bar{x} \in X_{0}$ with the property

$$
F_{\mu}(A x+t A(\bar{x}-x)) \geq F_{\mu}(A x)+c t, \quad \text { for all } t \in[0,1] .
$$

Then, $\psi_{V}$ is upper semicontinuous at $\mu$ with respect to the metric $d_{K}$ on $\mathscr{P}\left(\mathbb{R}^{s}\right)$, and there exist constants $L>0, \delta>0$ such that $\psi_{v}(v)$ is a CLM set for (1) and

$$
\left|\varphi_{V}(\mu)-\varphi_{V}(v)\right| \leq L d_{K}(\mu, v),
$$

whenever $d_{K}(\mu, v)<\delta, v \in \mathscr{P}\left(\mathbb{R}^{s}\right)$. 
Proof. Once more, we apply Theorem 2.1. We merely have to check whether the mapping $p \mapsto C_{p}(\mu)$ is pseudo-Lipschitzian at each $\left(x_{0}, p_{0}\right) \in$ $\psi_{v}(\mu) \times\left\{p_{0}\right\}$.

Let $x_{0} \in \psi_{\nu}(\mu)$, and consider at first the case where $F_{\mu}\left(A x_{0}\right)>p_{0}$. Then, there exists $\delta_{0}>0$ such that $F_{\mu}\left(A x_{0}\right)>p_{0}+\delta_{0}$; and, due to the continuity of $F_{\mu}$, we have $\epsilon_{0}>0$ such that

$$
F_{\mu}(A x) \geq p_{0}+\delta_{0}, \quad \text { for all } x \in B\left(x_{0}, \epsilon_{0}\right) .
$$

Hence,

$$
C_{p}(\mu) \cap B\left(x_{0}, \epsilon_{0}\right) \subseteq C_{p+\delta}(\mu), \quad \text { for each } p \in\left(p_{0}-\delta_{0}, p_{0}\right],
$$

and each $\delta \in\left(0, \delta_{0}\right)$. Therefore, the multifunction $p \mapsto C_{p}(\mu)$ is pseudo-Lipschitzian at $\left(x_{0}, p_{0}\right)$. Now, let $F_{\mu}\left(A x_{0}\right)=p_{0}$. Take $\epsilon_{0}>0$ and $c>0$ according to the assumption, and define $\delta_{0}:=c$ and $L:=c^{-1}$. We will show that

$$
C_{p}(\mu) \cap B\left(x_{0}, \epsilon_{0}\right) \subseteq C_{p+\delta}(\mu)+L \delta B_{m},
$$

for each $p \in\left(p_{0}-\delta_{0}, p_{0}\right]$ and each $\delta \in\left(0, \delta_{0}\right)$, which yields the desired pseudoLipschitzian property.

Let $p \in\left(p_{0}-\delta_{0}, p_{0}\right], \delta \in\left(0, \delta_{0}\right)$, be chosen arbitrarily, and consider $x \in C_{p}(\mu) \cap B\left(x_{0}, \epsilon_{0}\right)$. Due to the assumption, there exists $\bar{x} \in X_{0}$ such that (8) holds. In view of the convexity of $X_{0}$, without loss of generality, it is possible to select this $\bar{x}$ in such a way that we additionally have $\|\bar{x}-x\| \leq 1$. Consider

$$
y:=x+\delta c^{-1}(\bar{x}-x) \in X_{0} .
$$

Now,

$$
\|x-y\| \leq L \delta
$$

and

$$
F_{\mu}(A y)=F_{\mu}\left(A x+\delta c^{-1} A(\bar{x}-x)\right) \geq F_{\mu}(A x)+c \delta c^{-1} \geq p+\delta .
$$

Hence,

$$
y \in C_{p+\delta}(\mu) \text { and } x \in C_{p+\delta}(\mu)+L \delta B_{m} .
$$

The assertion finally follows from Theorem 2.1 .

Remark 2.3. If $F_{\mu}$ is continuously differentiable at $A x_{0}$, then (8) implies the constraint qualification used in Proposition 2.1.

Remark 2.4. Corollary 2.2 is a generalization of Corollary 2.1 when $X$ is given as in (7). 
To see this, suppose that $\mu \in \mathscr{M}_{r}$, for some $r \in(-\infty, 0]$, and assume that there exists $\bar{x} \in X_{0}$ such that $F_{\mu}(A \bar{x})>p_{0}$ (Slater condition). Then, the distribution function $F_{\mu}$ is continuous, since $\mu$ is absolutely continuous with respect to the Lebesgue measure on $\mathbb{R}^{s}$ (Ref. 27). Now, let $x_{0} \in X_{0}$ such that $F_{\mu}\left(A x_{0}\right)=p_{0}$. There exist $\epsilon_{0}>0$ and $\delta_{0}>0$ such that

$$
0<p_{0}-\delta_{0} \leq F_{\mu}(A x) \leq p_{0}+\delta_{0}<F_{\mu}(A \bar{x}), \quad \text { for all } x \in B\left(x_{0}, \epsilon_{0}\right) .
$$

We are going to show that, with a suitable $c>0$, condition (8) is fulfilled for any $x \in X_{0} \cap B\left(x_{0}, \epsilon_{0}\right)$.

For this, let without loss of generality $r<0$, and define

$$
a:=p_{0}-\delta_{0}>0 \text { and } b:=\left[F_{\mu}(A \bar{x})\right]^{r}-\left(p_{0}+\delta_{0}\right)^{r}<0 .
$$

We obtain, for arbitrary $t \in[0,1]$,

$$
\begin{aligned}
F_{\mu}(A x+t A(\bar{x}-x))^{r} & \leq t F_{\mu}(A \bar{x})^{r}+(1-t) F_{\mu}(A x)^{r} \\
& \leq a^{r}+t\left(F_{\mu}(A \bar{x})^{r}-\left(p_{0}+\delta_{0}\right)^{r}\right)=a^{r}+t b ;
\end{aligned}
$$

therefore,

$$
\begin{aligned}
F_{\mu}(A x+t A(\bar{x}-x)) & \geq\left(a^{r}+t b\right)^{1 / r} \\
& \geq a+t r^{-1} b a^{1-r}, \quad \text { for all } t \in\left[0,-a^{r} b^{-1}\right] .
\end{aligned}
$$

The last inequality holds, since the function $g(t):=\left(a^{r}+t b\right)^{1 / r}$ is convex for $t \in\left[0,-a^{r} b^{-1}\right]$; consequently,

$$
g(t) \geq g(0)+g^{\prime}(0) t, \quad \text { for } t \in\left[0,-a^{r} b^{-1}\right] .
$$

Taking finally into account that $-a^{r} b^{-1}>1$, we obtain (8) with $c:=r^{-1} b a^{1-r}$.

The following lemma is very useful when verifying the growth condition (8). Its proof is essentially based on an idea that has already been developed in Ref. 9, Lemma 4.9.

Lemma 2.1. Let $\mu \in \mathscr{P}\left(\mathbb{R}^{s}\right)$, let $X_{0}$ be a closed convex set, and fix some $x_{0} \in X_{0}$. Assume that $\mu$ has a density $f_{\mu}$ and that there exist $d>0, \rho>0$ such that

$$
f_{\mu}(z) \geq d, \quad \text { for all } z \in B\left(A x_{0}, \rho\right) .
$$

Furthermore, assume that there exists $\bar{x} \in X_{0}$ such that $A \bar{x} \geq A x_{0}$ and $A \bar{x} \neq A x_{0}$. Then, there exist $\epsilon_{0}>0$ and $c>0$ such that (8) holds for each $x \in X_{0} \cap B\left(x_{0}, \epsilon_{0}\right)$.

Proof. First, one confirms that, without loss of generality, it is possible to suppose $A \bar{x} \in B\left(A x_{0}, \rho_{1}\right)$ and $[A \bar{x}]_{1}>\left[A x_{0}\right]_{1}$, where $\rho_{1}:=\rho / 4$ and $[z]_{i}$ 
denotes the $i$ th component of $z \in \mathbb{R}^{s}$. Now, we choose $\epsilon_{0}>0$ such that, on the one hand, there exists $\xi \in \mathbb{R}$ such that

$$
[A \bar{x}]_{1}>\xi \geq[A x]_{1}, \quad \text { for all } x \in B\left(x_{0}, \epsilon_{0}\right),
$$

and on the other hand

$$
\max _{i=1, \ldots, s}\left|[A x]_{i}-\left[A x_{0}\right]_{l}\right| \leq \rho_{1}, \quad \text { for all } x \in B\left(x_{0}, \epsilon_{0}\right) \text {. }
$$

Denote

$$
a:=[A \bar{x}]_{1}-\xi>0 .
$$

Then, we have, for arbitrary $x \in X_{0} \cap B\left(x_{0}, \epsilon_{0}\right)$ and $t \in[0,1]$,

$$
\begin{aligned}
& F_{\mu}(A x+t A(\bar{x}-x))-F_{\mu}(A x) \\
& \geq \int_{[A x]_{1}}^{[A x]_{1}+t a} \int_{-\infty}^{[A x]_{2}} \cdots \int_{-\infty}^{[A x]_{s}} f_{\mu}\left(z_{1}, \ldots, z_{s}\right) d z_{s} \cdots d z_{1} \\
& \geq \int_{[A x]_{1}}^{[A x]_{1}+t a} \int_{\left[A x_{0}\right]_{2}-2 \rho_{1}}^{\left[A x_{0}\right]_{2}-\rho_{1}} \cdots \int_{\left[A x_{0}\right]_{s}-2 \rho_{1}}^{\left[A x_{0}\right]_{s}-\rho_{1}} f_{\mu}\left(z_{1}, \ldots, z_{s}\right) d z_{s} \cdots d z_{1} \\
& \geq t a \rho_{1}^{s-1} d .
\end{aligned}
$$

Hence, the desired result follows with

$$
c:=\left([A \bar{x}]_{1}-\xi\right) d(\rho / 4)^{s-1} .
$$

We remark that Corollaries 2.1 and 2.2 also represent qualitative stability results with respect to weak convergence of probability measures. This is mainly due to the smoothness assumptions imposed on the measures which led to $\mu$-uniformity classes (cf. Remark 2.1). On the other hand, also without such smoothness assumptions, conclusions from Theorem 2.1 may be drawn, as can be seen by the following remark where we deal with discrete distributions.

Remark 2.5. Let $\mu \in \mathscr{P}\left(\mathbb{R}^{s}\right)$ be a discrete measure with countable support; consider (1) with $X$ given by (7). Let $p_{0} \in(0,1)$ be such that

$$
\inf _{z \in \mathbb{R}^{s}}\left|F_{\mu}(z)-p_{0}\right|>0 \text {. }
$$

Then, there exists a neighborhood $U$ of $p_{0}$ such that

$$
C_{p_{0}}(\mu)=C_{p}(\mu), \quad \text { for all } p \in U ;
$$

consequently, the mapping $p \mapsto C_{p}(\mu)$ is pseudo-Lipschitzian at each $\left(x_{0}, p_{0}\right)$ with $x_{0} \in C_{p_{0}}(\mu)$. 
If the objective in (1) is Lipschitzian on bounded sets and if there exists a bounded open set $V \subset \mathbb{R}^{m}$ such that $\psi_{V}(\mu)$ is a CLM set for (1), we now obtain the stability assertions of Theorem 2.1 with respect to the Kolmogorov metric $d_{K}$.

In what follows, we indicate the potential of our general results for the situation of unknown distribution $\mu$. Let $\xi_{1}, \xi_{2}, \ldots$ be independent random variables on a probability space $(\Omega, \mathscr{A}, P)$ with values in $\mathbb{R}^{s}$ and common distribution $\mu$. Consider the empirical measure $\mu_{n}$ which is given by

$$
\mu_{n}(\omega):=n^{-1} \sum_{i=1}^{n} \delta_{\xi_{i}(\omega),}, \quad \omega \in \Omega, n \in \mathbb{N},
$$

where $\delta_{z} \in \mathscr{P}\left(\mathbb{R}^{s}\right)$ denotes the measure with unit mass at $z \in \mathbb{R}^{s}$. Then, it is known that (see, e.g., Ref. 25 and the references therein)

$$
\begin{aligned}
& d_{K}\left(\mu_{n}(\omega), \mu\right)=O\left((\log \log n / n)^{1 / 2}\right), \quad P \text {-almost surely, } \\
& P\left(\left\{\omega: d_{K}\left(\mu_{n}(\omega), \mu\right)>\epsilon\right\}\right) \leq C_{1} \exp \left(-C_{2} \epsilon^{2} n\right),
\end{aligned}
$$

where $C_{1}>0$ and $0<C_{2}<2$ are some constants. Inequality (10) is often referred to as the Dvoretzky-Kiefer-Wolfowitz inequality.

Our quantitative stability results together with relation (9) now give rise to rates for the almost-sure convergence of optimal values and optimal solutions if the unknown distribution $\mu$ is estimated by empirical distributions.

Let us finally illustrate how to combine our Lipschitz (or Hölder) stability results with Ineq. (10). Suppose for instance that we have a result of the type

$$
\left|\varphi_{V}(\mu)-\varphi_{v}(v)\right| \leq L d_{K}(\mu, v), \quad \text { whenever } d_{K}(\mu, v)<\delta ;
$$

see Corollary 2.2, Remark 2.5. Then, we obtain

$$
\begin{aligned}
& P\left(\left\{\omega:\left|\varphi_{V}\left(\mu_{n}(\omega)\right)-\varphi_{V}(\mu)\right|>\epsilon\right\}\right) \\
& \leq P\left(\left\{\omega: \epsilon<L d_{K}\left(\mu_{n}(\omega), \mu\right)\right\}\right)+P\left(\left\{\omega: d_{K}\left(\mu_{n}(\omega), \mu\right)>\delta\right\}\right) ;
\end{aligned}
$$

in view of $(10)$, we can continue,

$$
\leq 2 C_{1} \exp \left(-C_{2}(\min \{\epsilon / L, \delta\})^{2} n\right)
$$

Following the above way, in principle, it is possible to derive corresponding estimates for optimal solutions or feasible sets. In the latter case, one then arrives at results which are in the spirit of Theorem 3 and Proposition 1 in Ref. 14. 


\section{Application to Power Dispatch}

The problem of the optimal power dispatch consists of allocating amounts of electric power to generation units such that the total generation costs are minimal, while an electric power demand is met and certain additional constraints are satisfied. The generation units in our model are thermal power stations, pumped storage plants, and an energy contract. The generation process is considered for a time horizon up to one day with a discretization into hourly or half-hourly intervals. Unit commitment and network questions are excluded.

The special feature of our model is that randomness of electrical power demand is taken into account by formulating the equilibrium between total generation and demand as a chance constraint. The typical situation in practice, however, is that the probability distribution of the power demand is not completely available. In the present section, therefore, we apply the theory developed in Section 2 to derive sufficient conditions for the model to be stable under perturbations of the distribution of power demand. Before doing that, we introduce our model and give a few comments on possible model refinements.

Let $K$ and $M$ denote the number of thermal power stations and pumped storage plants. Let $N$ be the number of subintervals in the discretization of the time period. The unknown levels of production in the thermal power stations and the pumped storage plants are $y_{r}^{l}, i=1, \ldots, K$ and $r=1, \ldots, N$, $s_{r}^{j}, j=1, \ldots, M$ and $r=1, \ldots, N$ (generation mode), and $w_{r}^{j}, j=1, \ldots, M$ and $r=1, \ldots, N$ (pumping mode). By $z_{r}, r=1, \ldots, N$, we denote the unknown amounts of energy purchased or sold according to the contract.

The total generation costs are given by the fuel costs of the thermal power stations (which are assumed to be a strictly convex quadratic function of the generated power, cf. Refs. 21 and 22) plus the costs (respectively, takings) according to the energy contract (which are a linear function of the power). Concerning pumped storage plants, we remark that sometimes (Refs. 16 and 17) the stock in the upper dam is evaluated by a certain function such that another term enters the objective, which reffects the costs and takings, respectively, according to the change of stock caused by the operation of the plant. In our model, however, we do not pursue this; hence, the objective becomes

$$
y^{T} H y+h^{T} y+g^{T} z
$$

where $y \in \mathbb{R}^{K N}, z \in \mathbb{R}^{N}, H \in L\left(\mathbb{R}^{K N}, \mathbb{R}^{K N}\right)$ is positive definite and diagonal, $h \in \mathbb{R}^{K N}$, and $g \in \mathbb{R}^{N}$. 
According to the discretization of the time period, we have a demand vector $d$ of dimension $N$, which is understood as a random vector with distribution $\mu \in \mathscr{P}\left(\mathbb{R}^{N}\right)$. Claiming that a generation $(y, s, w, z)$ fulfills the demand with probability $p_{0} \in(0,1)$ then means that

$$
\mu\left(\left\{d \in \mathbb{R}^{N}: \sum_{i=1}^{K} y_{r}^{i}+\sum_{j=1}^{M}\left(s_{r}^{j}-w_{r}^{j}\right)+z_{r} \geq d_{r}, r=1, \ldots, N\right\}\right) \geq p_{0}
$$

In addition to this probabilistic constraint, we take into account conditions which characterize the operation of the different plants:

$$
\begin{aligned}
& \underline{a}_{1} \leq y \leq \bar{a}_{1}, \quad 0 \leq s \leq \bar{a}_{2}, \quad 0 \leq w \leq \bar{a}_{3}, \quad \underline{a}_{4} \leq z \leq \bar{a}_{4} ; \\
& S_{j}^{00}-S_{j}^{0} \leq \sum_{r=1}^{\tau}\left(s_{r}^{j}-\eta_{j} w_{r}^{j}\right) \leq S_{j}^{00}, \quad j=1, \ldots, M, \tau=1, \ldots, N ; \\
& \sum_{r=1}^{N}\left(s_{r}^{j}-\eta_{j} w_{r}^{j}\right)=b_{1 j}, \quad j=1, \ldots, M, \quad \sum_{r=1}^{N} z_{r}=b_{2} .
\end{aligned}
$$

Restrictions for the power output are modeled in (12). Inequalities (13) reflect the balance between generation and pumping (measured in energy) in the pumped storage plants; $S_{j}^{00}$ and $S_{j}^{0}$ denote, respectively, the initial and maximal stocks in energy in the upper dam. For each pumped storage plant, we assume that the maximal stock in water of the upper dam equals that of the lower dam and that no additional inflow or outflow occurs. We then put the pumping efficiency, denoted $\eta_{j}$, as the quotient of the energy that is gained when letting the full content of the upper dam go down and the energy that is needed when pumping the full content of the lower dam upward. A further refinement of the model is possible if the pumping efficiency is not put as a constant, but as a function of the actual stock in the upper dam (Ref. 36). Equations (14) are balances over the whole time period for the pumped storage plants and according to the energy contract, respectively. The model can be supplemented by further linear, nonprobabilistic constraints, for instance those reflecting fuel quotas in the thermal power stations.

Since we have different variables for generation and pumping in the pumped storage plants, our model does not exclude a priori the impracticable situation of simultaneous generation and pumping. However, it is possible to show that a generation $(y, s, w, z)$ which has, for some $j \in\{1, \ldots, M\}$ and 
some $r \in\{1, \ldots, N\}$, both $s_{r}^{j}>0$ and $w_{r}^{j}>0$ cannot be optimal. Altogether, our model can formally be expressed as

$$
\min \left\{f(x): x \in X_{0}, \mu\left(\left\{d \in \mathbb{R}^{N}: A x \geq d\right\}\right) \geq p_{0}\right\},
$$

where $x=(y, s, w, z) \in \mathbb{R}^{m}, m=N(K+2 M+1), f(x)$ is defined by (11), $X_{0} \subset \mathbb{R}^{m}$ is the bounded convex polyhedron given by (12)-(14), $A \in L\left(\mathbb{R}^{m}, \mathbb{R}^{N}\right)$ is a suitable matrix, and $\mu$ is the probability distribution of random demand.

The results of Section 2 now provide the following conclusions. If we assume that we have approached the true distribution of the demand sufficiently accurately, then the optimal production policies behave upper semicontinuously and the optimal costs are Lipschitz continuous if either (i), (ii), (iii) hold:

(i) we know that the true distribution has a certain convexity property [cf. (4)] and there exists a Slater point (Corollary 2.1);

(ii) the true distribution is a discrete one (Remark 2.5);

(iii) the true distribution has a density which is uniformly bounded below by a positive number on some neighborhood related to the set of optimal solutions and, among the optimal policies (with respect to the true distribution), there is no one which exhausts the full generation capacity; see the constraint (12), Corollary 2.2, Lemma 2.1. In practice, the latter requirement on the optimal generation policy is always fulfilled, since, due to lower demand during the night, there is usually at least one power station which, during at least one hour, does not work with maximum capacity.

An examination of the objective in the optimal power-dispatch model shows that it is possible to fulfill condition (6) with $q=2$ and $\left\|\left.x\right|_{*}:=\right\| y \|_{2}$; here, $\|\cdot\|_{2}$ is the Euclidean norm on $\mathbb{R}^{N K}$. Hence, in the presence of the assumptions made in Corollary 2.1, Remark 2.2 applies, and we have Hölder continuity (with exponent $1 / 2$ ) of the optimal generation policies in the thermal plants.

When the original distribution is estimated by empirical ones, then the presented stability results together with the considerations at the end of Section 2 yield rates of convergence for optimal values and optimal solutions.

Finally, let us point out that the material developed in Section 2 also applies to a number of practical models which are known from the literature (the STABIL model in Ref. 20, a flood control model in Ref. 37, and a model for water resources system planning in Ref. 38). 


\section{References}

1. Kall, P., Stochastic Linear Programming, Springer-Verlag, Berlin, Germany, 1976.

2. WeTs, R., Stochastic Programming: Solution Techniques and Approximation Schemes, Mathematical Programming: The State-of-the-Art 1982, SpringerVerlag, Berlin, Germany, pp. 566-603, 1983.

3. Klatte, D., A Note on Quantitative Stability Results in Nonlinear Optimization, Proceedings of the 19th Jahrestagung 'Mathematische Optimierung,' Edited by K. Lommatzsch, Humboldt-Universität, Berlin, Germany, pp. 77- 86, 1987.

4. Bank, B., Guddat, J., Klatte, D., Kummer, B., and TAmmer, K., Nonlinear Parametric Optimization, Akademie-Verlag, Berlin, Germany, 1982.

5. Robinson, S. M., Local Epi-Continuity and Local Optimization, Mathematical Programming, Vol. 37, pp. 208-223, 1987.

6. Kall, P., On Approximations and Stability in Stochastic Programming, Parametric Optimization and Related Topics, Edited by J. Guddat, H. Th. Jongen, B. Kummer, and F. Nožicka, Akademie-Verlag, Berlin, Germany, pp. 387-407, 1987.

7. WANG, J., Continuity of Feasible Solution Sets of Probabilistic Constrained Programs, Journal of Optimization Theory and Applications, Vol. 63, pp. 79-89, 1989.

8. Römisch, W., and Schultz, R., On Distribution Sensitivity in Chance Constrained Programming, Advances in Mathematical Optimization, Edited by $\mathrm{J}$. Guddat et al., Akademie-Verlag, Berlin, Germany, pp. 161-168, 1988.

9. RöMISCH, W., and WAKolbinger, A., Obtaining Convergence Rates for Approximations in Stochastic Programming, Parametric Optimization and Related Topics, Edited by J. Guddat, H. Th. Jongen, B. Kummer, and F. Nožicka, Akademie-Verlag, Berlin, Germany, pp. 327-343, 1987.

10. Römisch, W., and Schultz, R., Distribution Sensitivity in Stochastic Programming, Mathematical Programming, Vol. 50, pp. 197-226, 1991.

11. DupaČovã, J., Stability in Stochastic Programming-Probabilistic Constraints, Stochastic Optimization, Edited by V. I. Arkin, A. Shiraev, and R. Wets, Springer-Verlag, Berlin, Germany, pp. 314-325, 1986.

12. FIACCO, A. V., Introduction to Sensitivity and Stability Analysis in Nonlinear Programming, Academic Press, New York, New York, 1983.

13. Salinetti, G., Approximations for Chance-Constrained Programming Problems, Stochastics, Vol. 10, pp. 157-179, 1983.

14. Vogel, S., Stability Results for Stochastic Programming Problems, Optimization, Vol. 19, pp. 269-288, 1988.

15. Deák, I., Hoffer, J., Mayer, J., Németh, Á., Potecz, B., Prékopa, A., and StrazickY, B., Optimal Daily Scheduling of the Electricity Production in Hungary, Operations Research in Progress, Edited by G. Feichtinger and P. Kall, D. Reidel Publishing Company, New York, New York, pp. 103-114, 1982.

16. Gonzales, R., and Rofman, E., Computational Methods in Scheduling Optimization, Fermat Days 85: Mathematics for Optimization, Edited by J. B. HiriartUrruty, North-Holland, Amsterdam, Holland, pp. 135-155, 1986. 
17. Gonzales, R., and Rofman, E., On the Computation of Optimal Control Policies of Energy Production Systems with Random Perturbations, Proceedings of the 26th IEEE Conference on Decision and Control, Los Angeles, California, 1987.

18. HAPP, H. H., Optimal Power Dispatch-a Comprehensive Survey, IEEE Transactions on Power Apparatus and Systems, Vol. PAS-96, pp. 841-854, 1977.

19. Prekopa, A., Recent Results in Optimization of Electro-energetic Systems, Applied Optimization Techniques in Energy Problems, Edited by Hj. Wacker, Teubner, Stuttgart, Germany, pp. 354-383, 1985.

20. Prékopa, A., Ganczer, S., DeÁx, I., and PATYi, K., The STABIL Stochastic Programming Model and Its Experimental Application to the Electrical Energy Sector of the Hungarian Economy, Stochastic Programming, Edited by M. A. H. Dempster, Academic Press, London, England, pp. 369-385, 1980.

21. VAN DeN Bosch, P. P. J., Optimal Static Dispatch with Linear Quadratic and Nonlinear Functions of the Fuel Costs, IEEE Transactions on Power Apparatus and Systems, Vol. PAS-104, pp. 3402-3408, 1985.

22. VAN DEN Bosch, P. P. J., and LoOTSMA, F, A., Scheduling of Power Generation via Large-Scale Nonlinear Optimization, Journal of Optimization Theory and Applications, Vol. 55, pp. 313-326, 1987.

23. WACKER, Hj., Editor, Applied Optimization Techniques in Energy Problems, Teubner, Stuttgart, Germany, 1985.

24. Rockafellar, R. T., Lipschitzian Properties of Multifunctions, Nonlinear Analysis, Vol. 9, pp. 867-885, 1985.

25. Gaenssler, P., and Stute, W., Empirical Processes: A Survey of Results for Independent and Identically Distributed Random Variables, Annals of Probability, Vol. 7, pp. 193-243, 1979.

26. Bhattacharya, R. N., and Ranga RaO, R., Normal Approximation and Asymptotic Expansions, Wiley, New York, New York, 1976.

27. Borell, C., Convex Set Functions in d-space, Periodica Mathematica Hungarica, Vol. 6, pp. 111-136, 1975.

28. Prékopa, A., Logarithmic Concave Measures with Applications to Stochastic Programming, Acta Scientiarum Mathematicarum, Vol. 32, pp. 301-316, 1971.

29. Rinotr, Y., On Convexity of Measures, Annals of Probability, Vol. 4, pp. 1020 $1026,1976$.

30. Prékopa, A., Logarithmic Concave Measures and Related Topics, Stochastic Programming, Edited by M. A. H. Dempster, Academic Press, London, England, pp. 63-82, 1980 .

31. Johnson, N. L., and Kotz, S., Distributions in Statistics: Continuous Multivariate Distributions, Wiley, New York, New York, 1972.

32. RoBinson, S. M., Regularity and Stability for Convex Multivalued Functions, Mathematics of Operations Research, Vol. 1, pp. 130-143, 1976.

33. Pshenichny, B. N., Convex Analysis and Extremal Problems, Nauka, Moscow, USSR, 1980 (in Russian).

34. Robinson, S. M., Stability Theory for Systems of Inequalities, Part II: Differentiable Nonlinear Systems, SIAM Journal on Numerical Analysis, Vol. 13, pp. 497-513, 1976. 
35. Clarke, F. H., Optimization and Nonsmooth Analysis, Wiley, New York, New York, 1983.

36. Lindner, E., and WACKer, Hj., Input Parameters for the Optimization of the System Partenstein: Forecasting of the Influx Determination of the Efficiency Function, Applied Optimization Techniques in Energy Problems, Edited by $\mathrm{Hj}$. Wacker, Teubner, Stuttgart, Germany, pp. 286-320, 1985.

37. Prékopa, A., and SzÃntaI, T., Flood Control Reservoir System Design Using Stochastic Programming, Mathematical Programming Study, Vol. 9, pp. 138 $151,1978$.

38. Dupacová, J., Gatvoronski, A., Kos, Z., and Szántai, T., Stochastic Programming in Water Resources System Planning: A Case Study and a Comparison of Solution Techniques, Working Paper WP-86-40, IIASA, Laxenburg, Austria, 1986. 